\title{
Experimental Studies of the Developed Method for Determining the Insulation Parameters in an Asymmetric Network by a Voltage up to $1000 \mathrm{~V}$ at Mining Enterprises
}

\author{
A. B. Utegulov ${ }^{1, *}$, I. V. Koshkin ${ }^{2}$, and A. I. Koshkina ${ }^{2}$ \\ ${ }^{1}$ Energy Department, S. Seifullin Kazakh Agro-Technical University, 62 Pobeda avenue, Astana, Republic of Kazakhstan \\ ${ }^{2}$ Kostanay State University A. Baitursynov, Kostanay, Republic of Kazakhstan
}

\begin{abstract}
In the work experimental studies of the developed method for determining the insulation parameters in an asymmetric electrical network by a voltage up to $1000 \mathrm{~V}$ at mining enterprises were carried out. It is based on measuring the module values of line voltage, angle of phase shift between vectors of damaged phase voltage relative to the ground and line voltage at two other phases, as well on measuring the module value of damaged phase voltage relative to the ground before and after connecting additional capacitive conductivity between the network's phases and the ground. The results of experiment showed that the developed method for determining the insulation parameters has satisfactory accuracy and safety when operating three-phase electrical networks by voltage up to $1000 \mathrm{~V}$ at mining enterprises.
\end{abstract}

\section{Introduction}

In the three-phase electrical networks by voltage up to $1000 \mathrm{~V}$ a lack of phase voltages symmetry takes place. Asymmetry in low voltage networks is formed by two factors: asymmetric load on the phases of electrical network and if insulation of any phase relative to the ground is damaged.

State of insulation in an asymmetric electrical network with an isolated neutral (AENIN) by voltage up to $1000 \mathrm{~V}$ is characterized by its active conductivity that reduces insulation level in the network, as well as capacitive and active components of insulation's total conductivity.

It should be noted that active conductivity reducing the level of insulation (ACRLI) in a network with an isolated neutral by voltage up to $1000 \mathrm{~V}$ at mining enterprises characterizes the value of leakage current to the ground, as well as the value of touch voltage when the insulation is damaged. Therefore, in the practice of operating electrical installations it is necessary to know the value of ACRLI at one of electrical network's phases relative to the ground. By the value of ACRLI at one of electrical network's phases relative to the ground, organizational and technical measures are developed to increase the level of electrical safety in the AENIN by voltage up to $1000 \mathrm{~V}$ at mining enterprises [1].

To ensure an increase in the level of electrical safety, it is required to know the state of insulation on electrical installations under operating voltage. For this, it is necessary to develop methods for determining the ACRLI in the network, as well as the capacitive and active components of insulation's total conductivity in the three-phase AENIN by voltage up to $1000 \mathrm{~V}$. This will allow choose the right strategy for development of organizational and technical measures to increase the level of electrical safety in the networks by voltage up to $1000 \mathrm{~V}$ at mining enterprises.

\section{Theoretical basis of the developed method}

When developing a method for determining the ACRLI in the network by voltage up to $1000 \mathrm{~V}$ at mining enterprises under operating voltage, we use the method of determining the insulation parameters in a symmetric network with an isolated neutral by voltage up to $1000 \mathrm{~V}$ [1]. The method is based on: 1) measuring the module value of phase voltage relative to the ground before and after connecting additional active conductivity (AAC) between it and the ground, and measuring angle of phase shift between vectors of phase voltage relative to the ground and line voltage at two other phases for which no AAC is introduced relative to the ground;2) determining the insulation parameters taking into account the value of the introduced AAC.

To develop the method for determining the ACRLI in AENIN by voltage up to $1000 \mathrm{~V}$, we use the mathematical formula for determining the network's capacitive conductivity of insulation from [2]:

$$
b=\frac{U_{l} g_{z}\left(U_{l}-1,73 U_{p h z} \sin \alpha\right)}{U_{l}^{2}+3 U_{p h z}^{2}-3,46 U_{l} U_{p h z} \sin \alpha}
$$

where: $U_{p h z}$ - the phase voltage relative to the ground after connecting AAC between it and the ground; $U_{l}-$

*Corresponding author: utegulov arman@ mail.ru 
line voltage; $\alpha$ - angle of phase shift between vectors of the phase voltage relative to the ground and the line voltage at two other phases for which no AAC is introduced relative to the ground; $g_{z}-$ AAC, which is connected between one of the network's phases and the ground to create artificial asymmetry.

From equation (1) for determining the capacitive conductivity of insulation in the symmetric network with an isolated neutral by voltage up to $1000 \mathrm{~V}$, it follows that if the insulation is damaged, $g_{z}$ will be the ACRLI in AENIN by voltage up to $1000 \mathrm{~V}$. To determine the ACRLI in AENIN by voltage up to $1000 \mathrm{~V}$, we connect $b_{z}$ - additional capacitive conductivity (ACC) between network's phases and ground (wherein all the introduced conductivities per phase are equal).

When connecting ACC $\left(b_{z}\right)$ between the network's phases and ground, equation (1) takes the form:

$$
b+b_{z}=\frac{U_{l} g_{z}\left(U_{l}-1,73 U_{p h z 1} \sin \alpha_{1}\right)}{U_{l}^{2}+3 U_{p h z 1}^{2}-3,46 U_{l} U_{p h z 1} \sin \alpha_{1}}
$$

where: $U_{p h z 1}-$ phase voltage relative to the ground after connecting ACC between the network's phases and the ground; $\alpha_{1}$ - angle of phase shift between vectors of the damaged phase voltage relative to the ground and the line voltage at two other phases, which are larger in value than the measured damaged phase voltage relative to the ground after connecting ACC between the network phases and the ground.

Solving equations (1) and (2) together, we obtain a mathematical formula for determining the ACRLI in AENIN by voltage up to $1000 \mathrm{~V}$ :

$$
g_{z}=\frac{\left(U_{l}^{2}-3 U_{p h z}^{2}-3,46 U_{l} U_{p h z} \sin \alpha\right) \cdot\left(U_{l}^{2}-3 U_{p h z 1}^{2}-3,46 U_{l} U_{p h z 1} \sin \alpha_{1}\right)}{U_{l}\left[\begin{array}{l}
\left(U_{l}^{2}-3 U_{p h z}^{2}-3,46 U_{l} U_{p h z} \sin \alpha\right) \cdot\left(U_{l}-1,73 U_{p h z 1} \sin \alpha_{1}\right)- \\
-\left(U_{l}^{2}-3 U_{p h z 1}^{2}-3,46 U_{l} U_{p h z 1} \sin \alpha_{1}\right) \cdot\left(U_{l}-1,73 U_{p h z} \sin \alpha\right)
\end{array}\right]} b_{z}
$$

From the obtained equation (3), it follows that the developed method for determining the ACRLI in AENIN by voltage up to $1000 \mathrm{~V}$, is based on measuring: 1) the module value of line voltage; 2) the angle of phase shift between vectors of the damaged phase voltage relative to the ground and the line voltage vector at two other phases; 3) the damaged phase voltage relative to the ground before and after connecting the ACC between the network's phases and the ground.

When developing a method for determining the capacitive conductivity of insulation in the AENIN by voltage up to $1000 \mathrm{~V}$ at mining enterprises under operating voltage, we use a method for determining the insulation parameters in a symmetric network with an isolated neutral by voltage up to $1000 \mathrm{~V}$ [2].

To determine the capacitive conductivity of insulation in the AENIN by voltage up to $1000 \mathrm{~V}$, in equation (1) we substitute the value of ACRLI obtained in equation (3). Solving equations (1) and (3) together, we obtain a mathematical formula for determining the capacitive conductivity of insulation in the AENIN by voltage up to $1000 \mathrm{~V}$ :

$$
\begin{aligned}
b= & \frac{\left(U_{l}^{2}-3 U_{p h z 1}^{2}-3,46 U_{l} U_{p h z 1} \sin \alpha_{1}\right) \cdot\left(U_{l}-1,73 U_{p h z} \sin \alpha\right)}{\left(U_{l}^{2}-3 U_{p h z}^{2}-3,46 U_{l} U_{p h z} \sin \alpha\right) \cdot\left(U_{l}-1,73 U_{p h z 1} \sin \alpha_{1}\right)-} b_{z} \\
& -\left(U_{l}^{2}-3 U_{p h z 1}^{2}-3,46 U_{l} U_{p h z 1} \sin \alpha_{1}\right) \cdot\left(U_{l}-1,73 U_{p h z} \sin \alpha\right)
\end{aligned}
$$

To develop a method for determining the total conductivity of insulation in the AENIN by voltage up to $1000 \mathrm{~V}$ at mining enterprises under operating voltage, we use a method to determine the insulation parameters in a three-phase electrical network with an isolated neutral by voltage above $1000 \mathrm{~V}$ [3]

The method for determining the insulation parameters in a three-phase electric network with an isolated neutral by voltage above $1000 \mathrm{~V}$ is based on measuring the module values of line voltage and phase voltage relative to the ground after the AAC is connected between it and the ground. Then taking into account the value of introduced AAC are determined [3]:

- total conductivity of network's insulation

$$
y=U_{p h z} U_{z}^{-1} g_{z}
$$

- active conductivity of network's insulation

$$
g=0,5 U_{z}^{-2}\left(U_{l}^{2}-3 U_{p h z}^{2}-U_{z}^{2}\right) g_{z}
$$

where $U_{z}$ - zero sequence voltage; $g_{z}-\mathrm{AAC}$, which is introduced between the phase of electrical network and the ground.

Solving equations (3) and (5) together, we obtain the formula for determining the total conductivity of insulation in the AENIN by voltage up to $1000 \mathrm{~V}$ :

$$
y=\frac{U_{p h z} \cdot\left(U_{l}^{2}-3 U_{p h z}^{2}-3,46 U_{l} U_{p h z} \sin \alpha\right)^{0.5} \cdot\left(U_{l}^{2}-3 U_{p h l}^{2}-3,46 U_{l} U_{p h l} \sin \alpha_{1}\right)}{U_{l}\left[\begin{array}{l}
\left(U_{l}^{2}-3 U_{p h z}^{2}-3,46 U_{l} U_{p h z} \sin \alpha\right) \cdot\left(U_{l}-1,73 U_{p h l} \sin \alpha_{1}\right)- \\
-\left(U_{l}^{2}-3 U_{p h l z}^{2}-3,46 U_{l} U_{p h l} \sin \alpha_{1}\right) \cdot\left(U_{l}-1,73 U_{p h z} \sin \alpha\right)
\end{array}\right]} b_{z}
$$

To develop a method for determining the active conductivity of insulation in the AENIN by voltage up to $1000 \mathrm{~V}$ at mining enterprises under operating voltage, solving equations (3) and (6) together, we obtain the formula for determining the active conductivity of network's insulation:

$$
g=\frac{1,73 U_{l} U_{p h z} \sin \alpha\left(U_{l}^{2}-3 U_{p h z 1}^{2}-3,46 U_{l} U_{p h z 1} \sin \alpha_{1}\right)}{U_{l}\left[\begin{array}{l}
\left(U_{l}^{2}-3 U_{p h z}^{2}-3,46 U_{l} U_{p h z} \sin \alpha\right) \cdot\left(U_{l}-1,73 U_{p h z 1} \sin \alpha_{1}\right)- \\
-\left(U_{l}^{2}-3 U_{p h z 1}^{2}-3,46 U_{l} U_{p h z 1} \sin \alpha_{1}\right) \cdot\left(U_{l}-1,73 U_{p h z} \sin \alpha\right)
\end{array}\right]} b_{z}
$$

The developed method provides simplicity and satisfactory accuracy when determining insulation parameters in an asymmetric network with an isolated neutral by voltage up to $1000 \mathrm{~V}$ at mining enterprises.

\section{Experimental studies of the developed method at laboratory conditions}

For practical purposes, a comparative analysis of errors is required when conducting experimental studies of the developed method and ammeter-voltmeter method, elaborated by Professor L.V. Gladilin [1]. 
Determination of insulation parameters by the ammeter-voltmeter method consists in measuring the module value of phase voltage relative to the ground before and after connecting AAC and measuring the value of a single phase ground fault current by directly connecting the phase of electrical installation to the ground.

Based on measurements of the module value of phase voltage relative to the ground before and after connecting $\mathrm{AAC}-U_{p h}$ and $U_{p h z}$ respectively, the module value of a single phase ground fault current $-I_{z}$, also taking into account the value of AAC $-g_{z}$, insulation parameters are determined by the formulas:

- total conductivity of network's insulation

$$
y=I_{z} U_{p h}^{-1}
$$

- summarized conductivity of network's insulation

$$
y_{\Sigma}=I_{z} U_{p h z}^{-1}
$$

- active conductivity of network's insulation

$$
g=0,5\left(y_{\Sigma}^{2}-y^{2}-g_{1}^{2}\right) 2 g_{z}^{-1}
$$

- capacitive conductivity of network's insulation

$$
b=\left(y^{2}-g^{2}\right)^{0,5}
$$

According to the measured values of line voltage $U_{l}$, phase voltages relative to the ground $-U_{p h z}$ and $U_{p h z l}$, angle of phase shift between vectors of the line voltage and the phase voltage relative to the ground $-\alpha$ and $\alpha_{1}$ before and after connecting $\mathrm{ACC}-b_{z}$ between the phases of electrical installation and the ground, also taking into account the value of ACC, according by mathematical dependencies (3), (4), (7) and (8) is determined: 1$)$ the active conductivity $-g_{z}$, reducing the level of insulation between one of the phases of electrical network and the ground; 2) capacitive, active and total conductivity of network's insulation relative to the ground.

To conduct experimental studies of the ammetervoltmeter method and the developed method, a distribution electrical network scheme at laboratory complex "Fundamentals of electrical safety" OEB1-CP26.08.2005 was built (Fig. 1).

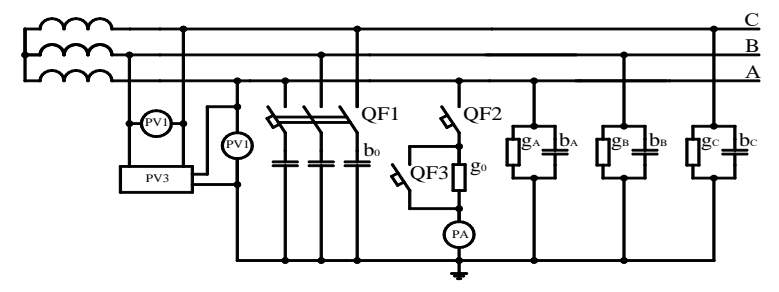

Fig. 1.Distribution electrical network scheme of the study of the ammeter-voltmeter method and the developed method.
To measure the module values of current and voltage, devices of type E-515 with an accuracy class of 0.5 and with current measurement limits $I=0 \div 5 \mathrm{~A}$, voltage measurement limits $U=0 \div 500 \mathrm{~V}$ were used. Resistance type PE-200 with nominal value $R_{z}=1000$ Ohms as the AAC was used. Capacitors type KBGS-MP with a capacity of $0.5 \mu \mathrm{F}$ and nominal voltage $U_{n}=$ $1000 \mathrm{~V}$ as the ACC were used.

The credibility study of the developed method in comparison with the ammeter-voltmeter method was carried out according to the following program.

1. Three circuit breakers QF1, QF2 and QF3 were selected.

2.Testing for operability of the selected circuit breakers was carried out.

3. After checking the circuit breakers QF1, QF2 and QF3 for operability, work on preparation of connection circuits for $\mathrm{ACC}-b_{z}$ and $\mathrm{AAC}-g_{z}$ was produced.

4. The circuit breaker QF1 was connected to phases $\mathrm{A}, \mathrm{B}$ and $\mathrm{C}$ of the electrical network. For switching between outgoing contacts of the circuit breaker and ground the ACC $\left(b_{z}\right)$ was connected.

5. To phase $\mathrm{A}$ of the circuit breaker QF2 was connected the AAC $\left(g_{z}\right)$ that reduces the insulation resistance between this phase and the ground. AAC can be shunted by the circuit breaker QF3. Between the AAC $\left(g_{z}\right)$ and the ground the ammeter PA was connected.

6. After connecting circuit breakers $(\mathrm{QF} 1, \mathrm{QF} 2$ and QF3), ACC $\left(b_{z}\right)$, AAC $\left(g_{z}\right)$, ammeter (PA) to the electrical network, measuring devices PV1, PV2 and PV3 were connected. They measured the module values of the line voltage, phase voltage relative to ground and the angle of phase shift between vectors of the phase voltage relative to ground and the line voltage.

7. After work on item 6 had been done the values of connected for the experimental study measuring devices were recorded.

8. After work on item 7 had been done, by the circuit breaker QF2 the AAC was connected, and the current flowing through this conductivity with the ammeter RA was recorded. By voltmeters PV1 and PV2 the module values of line voltage, phase voltage relative to the ground were measured. By the measuring device PV3 the angle of phase shift between vectors of the phase voltage relative to the ground and the line voltage was recorded.

9. After work on item 8 had been done, by the circuit breaker QF3 the AAC $\left(g_{z}\right)$ between the network phase and the ground was shunted. The value of current flowing without AAC with the ammeter PA was recorded. Also measurement work on item 8 was conducted.

10. After work on item 9 had been done, the circuit breaker QF3 was turned off, thereby the AAC $\left(g_{o}\right)$ between the network phase and the ground was connected. The value of current flowing through the AAC with the ammeter PA was recorded. Also measurement work on item 8 was conducted. 
Table 1.The results of determining the insulation parameters by the method of ammeter-voltmeter.

\begin{tabular}{|l|c|c|c|c|c|c|c|c|}
\hline \multicolumn{1}{|c|}{ Insulation parameters } & \multicolumn{9}{|c|}{ Number of measurements } \\
\cline { 2 - 9 } & $\mathbf{1}$ & $\mathbf{2}$ & $\mathbf{3}$ & $\mathbf{4}$ & $\mathbf{5}$ & $\mathbf{6}$ & $\mathbf{7}$ & $\mathbf{8}$ \\
\hline Total conductivity of network insulation $y \times 10-3, \mathrm{~S}$. & 0,80 & 0,75 & 0,75 & 0,72 & 0,75 & 0,68 & 0,71 & 0,86 \\
\hline Capacitive conductivity of network insulation $\boldsymbol{b} \times 10-3, \mathrm{~S}$. & 0,68 & 0,77 & 0,83 & 0,48 & 0,69 & 0,58 & 0,75 & 0,62 \\
\hline Active conductivity network insulation $\mathrm{g} \times 10-3, \mathrm{~S}$. & 0,32 & 0,21 & 0,34 & 0,32 & 0,37 & 0,28 & 0,49 & 0,27 \\
\hline
\end{tabular}

Table 2.The results of determining the insulation parameters by the developed method.

\begin{tabular}{|l|c|c|c|c|c|c|c|c|}
\hline \multicolumn{1}{|c|}{ Insulation parameters } & \multicolumn{9}{|c|}{ Number of measurements } \\
\cline { 2 - 9 } & $\mathbf{1}$ & $\mathbf{2}$ & $\mathbf{3}$ & $\mathbf{4}$ & $\mathbf{5}$ & $\mathbf{6}$ & $\mathbf{7}$ & $\mathbf{8}$ \\
\hline Total conductivity of network insulation $y \times 10-3, \mathrm{~S}$. & 0,84 & 0,69 & 0,75 & 0,71 & 0,85 & 0,88 & 0,83 & 0,75 \\
\hline Capacitive conductivity of network insulation $\boldsymbol{b} \times 10-3, \mathrm{~S}$. & 0,68 & 0,77 & 0,71 & 0,65 & 0,87 & 0,73 & 0,91 & 0,65 \\
\hline Active conductivity network insulation $\mathrm{g} \times 10-3, \mathrm{~S}$. & 0,25 & 0,32 & 0,32 & 0,35 & 0,25 & 0,37 & 0,36 & 0,35 \\
\hline
\end{tabular}

Table 3.Probabilistic-statistical characteristics of the insulation parameters of the electrical network.

\begin{tabular}{|l|c|c|c|c|c|}
\hline \multicolumn{1}{|c|}{ Method of measurement } & Parameters & $\bar{X}, 10^{-3} \mathrm{~S}$ & $S_{n}, 10^{-3} \mathrm{~S}$ & $\Delta X, 10^{-3} \mathrm{~S}$ & $\Delta X_{*}, \%$ \\
\hline \multirow{3}{*}{ By the method of ammeter-voltmeter } & $y$ & 0,743 & 0,042 & 0,040 & 2,2 \\
& $b$ & 0,775 & 0,037 & 0,032 & 3,5 \\
\hline \multirow{3}{*}{ By the developed method } & $g$ & 0,300 & 0,010 & 0,079 & 2,4 \\
& $y$ & 0,804 & 0,006 & 0,007 & 0,4 \\
& $b$ & 0,745 & 0,005 & 0,005 & 0,3 \\
\hline
\end{tabular}

11. After work on item 10 had been done, by the circuit breaker QF1 the ACC $\left(b_{z}\right)$ between the network's phases and the ground were connected. The value of current flowing through the AAC with the ammeter PA was recorded. Also measurement work on item 8 was conducted.

12. After work on item 11 had been done, the circuit breakers QF1 and QF2 were turned off.

With a time interval of 0.5 hours, the work on items 8 $\div 12$ alternately was performed. After work with a time interval of 0.5 hours and number of measurements $n=8$ had been done, the distribution electrical network scheme for the study of methods for determining the insulation parameters in the AENIN by voltage up to $1000 \mathrm{~V}$ was dismantled.

To compare the developed method with the classical method of an ammeter-voltmeter the results of experimental studies were recorded in tables 1 and 2.

To prove credibility, according to estimation of errors set out in $[4,5]$, it is necessary to make at least four measurements. In this case, we took eight measurements with a time interval of 0.5 hours.

To compare the results of the developed method and the ammeter-voltmeter method, error estimation was produced [4, 5] and the following parameters are determined (obtained results are summarized in table 3):

1. Average values $\bar{X}$ of the studied parameters.

2. Mean square deviations of a single result $\sigma$ at $\mathrm{n}=$ 8 measurements of the studied parameter value.

3. Mean square error of a single result $S_{n}$ of the studied parameter.

4. An absolute error $\Delta X$ in determining the desired quantities.
5. A relative error $\Delta X_{*}$ of the compared methods for determining the insulation parameters of the electrical network's phases relative to the ground.

An analysis of the foregoing measurement errors allows us to conclude that the developed method for determining the insulation parameters can be used along with the method of ammeter-voltmeter. The proposed method with sufficient safety during operation can be recommended for implementation in production.

\section{Conclusions}

The following results are solved in the work:

1.A method for determining the insulation parameters in an asymmetric network by voltage up to $1000 \mathrm{~V}$ was developed. It based on measuring: 1) the module value of line voltage; 2) the angle of phase shift between vectors of the damaged phase voltage relative to the ground and the line voltage at two other phases; 3 ) damaged phase voltage relative to the ground before and after connecting the additional capacitive conductivity between the electrical network's phases and the ground.

2. A technique for an experimental study of the insulation state in a three-phase asymmetric network with an isolated neutral by voltage up to $1000 \mathrm{~V}$ was developed. The technique provides satisfactory accuracy, simplicity, and safety of work in existing electrical installations with voltage up to $1000 \mathrm{~V}$.

3. The comparative characteristics of the insulation parameters of the electrical network obtained as a result of the experiment showed that the developed method for determining the insulation parameters has satisfactory accuracy, since the mean square errors of single measurements and the relative mean square errors of 
determining the insulation parameters of electrical networks in the developed method and the ammetervoltmeter method are practically the same.

\section{References}

[1] L.V. Gladilin, V.I. Schutsky, Yu.G. Batsezhev, N.I. Chebotaev, Electrical Safety in the Mining Industry (Moscow, Nedra, 1977) 327

[2] B.B. Utegulov, The Method for Determining the Insulation Parameters in an Asymmetric Network with a Voltage of Up to $1000 \mathrm{~V}$ (Vladivostok, International Multi-Conference FarEastCon, 2019)

[3] V.I. Schutsky, B.B. Utegulov, The method for determining the insulation parameters of threephase electrical networks with an isolated neutral by voltage above 1000 V, U.S.S.R. Copyright certificate 917127, MKI G01R27/18, Bulletin 12 (30 March 1982)

[4] P.K. Khromoin, Electrotechnical measurements Textbook (Moscow, Forum, 2013) 288

[5] B.B. Utegulov, A.B. Utegulov, I.V. Koshkin, A.I. Koshkina, The influence of some natural factors on efficiency of operation of photoelectric elements under conditions of North Kazakhstan, IOP Conference Series: Materials Science and Engineering, Saint Petersburg (2019) 